\title{
DESIGN CONSIDERATIONS FOR DEVELOPING BIODEGRADABLE MAGNESIUM IMPLANTS
}

\author{
Harpreet S. Brar ${ }^{1}$, Benjamin G. Keselowsky ${ }^{2}$, Malisa Sarntinoranont ${ }^{3}$, Michele V. Manuel ${ }^{1}$ \\ ${ }^{1}$ University of Florida, Department of Materials Science and Engineering; Gainesville, FL 32611, USA \\ ${ }^{2}$ University of Florida, Department of Biomedical Engineering; Gainesville, FL 32611, USA \\ ${ }^{3}$ University of Florida, Department of Mechanical and Aerospace Engineering; Gainesville, FL 32611, USA
}

Keywords: Bioabsorbable, Design, Magnesium Alloy

\begin{abstract}
The integration of biodegradable and bioabsorbable magnesium implants into the human body is a complex undertaking that faces major challenges. The complexity arises from the fact that biomaterials must meet both engineering and physiological requirements to ensure the desired properties. Historically, efforts have been focused on the behavior of commercial magnesium alloys in biological environments and their resultant effect on cell-mediated processes. Developing causal relationships between alloy chemistry and microstructure, and its effect on cellular behavior can be a difficult and time intensive process. A systems design approach driven by thermodynamics has the power to provide significant contributions in developing the next generation of magnesium alloy implants with controlled degradability, biocompatibility, and optimized mechanical properties, at reduced time and cost. This approach couples experimental research with theory and mechanistic modeling for the accelerated development of materials. The aim of this article is to enumerate this strategy, design considerations and hurdles for developing new magnesium alloys for use as biodegradable implant materials [1].
\end{abstract}

\section{Reference}

[1] H.S. Brar, B.G. Keselowsky, M. Sarntinoranont, M.V. Manuel, "Design Considerations for Developing Biodegradable Magnesium Implants", JOM 63/4 (2011), to be published. 